\title{
Application of the Mechanism Design to Develop the RGB LEDs Color Mixing
}

\author{
Chih-Ching Hung, ${ }^{1}$ Yan-Huei Li, ${ }^{2}$ and Ping-Han Yang ${ }^{1}$ \\ ${ }^{1}$ Department of Product Design, Shu-Te University, Kaohsiung 82445, Taiwan \\ ${ }^{2}$ Department of the Mechanical and Automation Engineering, National Kaohsiung First University of Science and Technology, \\ Kaohsiung, Taiwan
}

Correspondence should be addressed to Chih-Ching Hung; hungcc16@stu.edu.tw

Received 28 February 2015; Accepted 15 April 2015

Academic Editor: Ahmad Umar

Copyright (C) 2015 Chih-Ching Hung et al. This is an open access article distributed under the Creative Commons Attribution License, which permits unrestricted use, distribution, and reproduction in any medium, provided the original work is properly cited.

\begin{abstract}
The big advantage of LED is the flexible spectral design to make white light using different color mixing schemes. Recently, the color mixing of RGB LEDs is mostly done by sealing all three chips at single package and regulated the mix ratio of these three colors to produce the color of light. And, by changing the LEDs array alignment, RGB chips can achieve overlapping colors of light and create the fun of changing colors in light mixing. Therefore, the purpose of this study is to propose an innovative technique of light mixing. By applying the mechanism design, a RGB light mixing mechanism is produced. Each of the RGB LEDs lamp-type is installed on the couple link of the three mechanisms, respectively. By driving a crank makes the couple link and an output link to produce the relative motion, this will result in the fact that the RGB lamps can project lights on the same plane in order to obtain the color mixing. Unlike mixing technique by control system, this design generates light mixing and changes of color with the synchronized driving of three mechanisms, thus achieving the dazzling perception of single-color lights or mixing of multiple colors for creating ambience of a space.
\end{abstract}

\section{Introduction}

Human sensation is caused by the perception of external stimuli and consists of five senses: visual, auditory, olfactory, taste, and tactile, where the visual sense constitutes $80 \%$ of the entire human perception, followed by auditory, tactile, olfactory, and taste sense [1]. Therefore, the eyes are the most important organ that people receive information directly from the outside world. The colors people see in the real life are the visual perception generated by lights reflected from objects into their eyes and transmitted to the nerves in the brain. As a result, colors are the nonverbal and visual way of the information transmission. Thus, visual, color, and illumination are tightly bonded together.

The changes in human lifestyle make people focus their attention on indoor lighting fixture design that generates comfortable ambience, such as the rotating neon light that creates a colorful ambience in the room. The designs evolved from color filter lenses in the old days to LEDs of today [2], as shown in Figure 1. Therefore, what an ambience-creating lighting fixture does is to present multiple colors in a space with, for example, gradual or direct change of colors. In recently years, light-emitting diodes (LEDs) are widely used in variant sources of lighting. With the rapid development in technology, LED has become bright and even more efficiency than conventional light sources in our environment [3]. Light-emitting diodes (LEDs) are penetrating into the huge market of general lighting because they are energy saving and environmentally friendly. The big advantage of LED light sources, compared to traditional incandescent lamps and fluorescent light tubes, is the flexible spectral design to make white light using different color mixing schemes. The spectral design flexibility of white LED light sources will promote them for novel applications to improve the life quality of human beings [4].

Since LEDs become increasingly common in recent times, users can find new applications for LED light, which provides unique features such as color tunable illumination. 




(a) Traditional color filter lenses
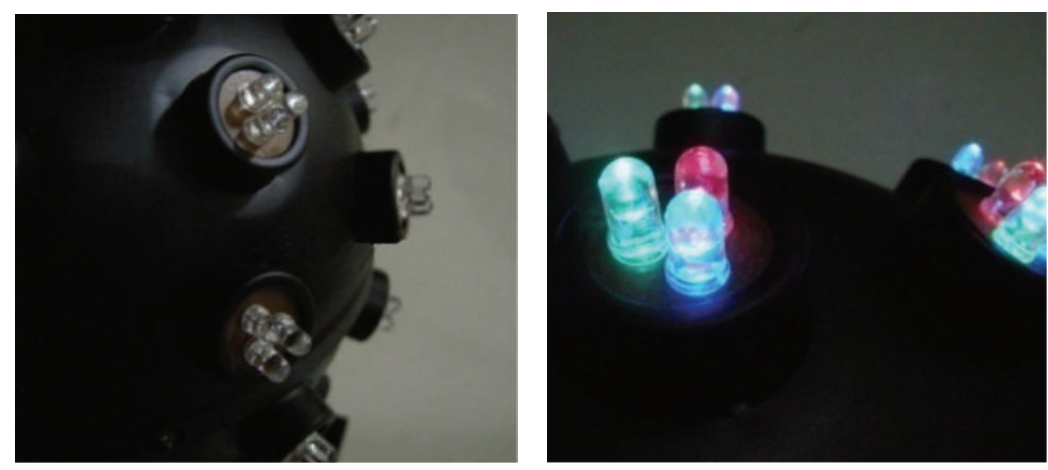

(b) LED light source

FIGURE 1: Rotating neon light [2].

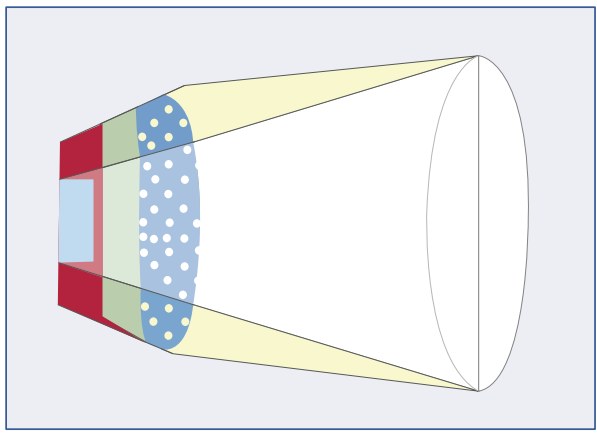

(a) UV LED + RGB phosphor

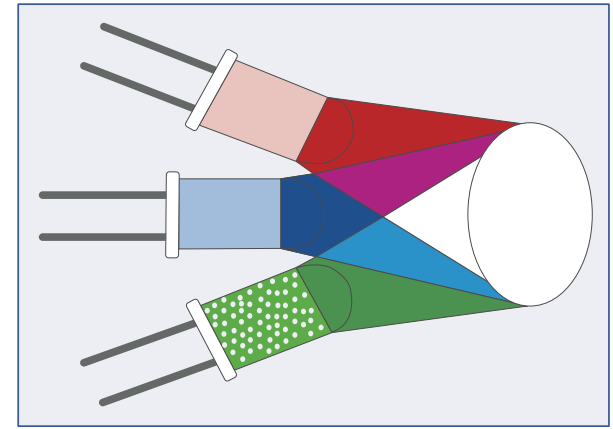

(b) Red + green + blue LEDs

FIGURE 2: Two methods of generating light from LEDs [7].

The color of illumination is easily tuned because LED sources may be integrated with small color LED chips, and their color output may be manipulated without inefficient filters. The color controllability of LEDs is very attractive in applications as down lighting, spot lighting, entertainment, architectural, floodlight, and show lighting, where a narrow color beam is projected at a distance $[5,6]$. Multicolor LEDs offer realtime control of its color emission as never before in lighting history. Therefore, the controllability is maximized by the ability of LED lamp to have their light output manipulated without inefficient color filters. There are two distinct approaches for creating mixed light with LEDs, shown in Figure 2. The first is combining blue LEDs with a down conversion phosphor. The second is mixing monochromatic (RGB) LEDs in appropriate proportions [7]. In lighting application, RGB LEDs can provide more color selection in correlated color temperature (CCT) and color rendering [8]. Thus, color mixing with RGB LEDs is an important issue in not only display but also in lighting application. The RGB LEDs approach allows for the facile dynamic control of color points and provides high color rendition and stabilization of the chromaticity. Therefore, the RGB LEDs have the good color rendering and illumination efficiency to exhibit the true color for plate display [9-12]. Hashimoto et al. [13] proposed the LED light source, especially composed of $R, G$, and B LED chips, having strong possibilities with high feeling of contrast, because these LED light sources have narrowband type spectral power distributions different from those of conventional light sources. And RGB LEDs were shown to have sufficiently narrow emission bands for composing them into trichromatic lighting clusters with a high ability of saturation of surface colors [14]. Lee [15] investigated the influence of the alignment of RGB LEDs on the uniformity of color and proposed the alignment of RG-B-RG to improve the color uniformity in the backlight module of monitor. Grabovičkić et al. [16] proposed a metal-less RXI collimator. The collimator's back surface is designed as a grooved surface providing two TIR reflections for all rays impinging on it. One advantage of the design is the lower manufacturing cost, since there is no need for the expensive process of metalization. Hsieh et al. [17] illustrate the light superposition effect analysis to achieve the light mixing by combining multiple LED modules. These modules can make the correct light pattern creative. These designs perform good color mixing, as well as being very insensitive to the source nonuniformities. Therefore, the light mixing using RGB LEDs is to place the $R, G$, and $B$ chips in single package and change the light flux of chips or output power by realigning the LED chips or regulating the voltage input to the chips, thus achieving the control of proportion of incident lights and achieving the 
purpose of light mixing. This allows the generation of light mixing effect of multiple colors with the color of light source [18-21].

The RGB LEDs provide many opportunities for illumination. One of these opportunities is to create a color tunable light source. This opens the field for applications as mood lighting [21]. Therefore, the adjustable LED lighting driven by multicolor sensor creates a harmonic and healthy natural lighting environment and in turn improves people's comfort, productivity, safety, and efficiency. However, the color mixing of RGB LEDs is mostly done by sealing all three chips at single package and regulated the mix ratio of these three colors to produce the color of light. The quantity of voltage and current inputted is used to control the RGB colors mix ratio. And, by changing the LEDs array alignment, RGB chips can achieve overlapping colors of light and create the fun of changing colors in light mixing [18-21]. Therefore, the purpose of this study is to propose an innovative technique of light mixing. The principles of mechanism design are introduced to the proposed technique. By applying the mechanism design, a RGB light mixing mechanism is produced. Each of the RGB LEDs lamp-type is installed on the couple link of the three four-bar mechanism, respectively. By driving a crank makes the couple link and an output link produce the relative motion; this will result in the RGB lamps where setup on the couple links can project lights on the same plane in order to obtain the color mixing. Unlike mixing technique by control system, this design generates light mixing and changes of color with the synchronized driving of three mechanisms and the rotating angle of the crank, thus achieving the dazzling perception of single-color lights or mixing of multiple colors and facilitating the need and selection for creating ambience of a space. This makes the lighting design much closer to the ergonomic design for human vision and accomplishes the purpose of cultural for science and technology product.

\section{Experiment}

2.1. Mechanism Design. A mechanism is a mechanical device that has the purpose of transferring motion and/or force from a source to an output. A linkage consists of links, generally considered rigid, which are connected by joints. Mechanisms are used in a great variety of machines and devices. The simple closed-loop linkage is the four-bar linkage, which has three moving links, one fixed link, and four revolute joints [22], shown in Figure 3. The four-bar linkage included fixed link (link 1), input link (link 2), couple link (link 3), follower link (link 4), and four revolute joints (joint a, b, c, and d). The link that is connected to the power source is called the input link. The follower link connected the moving pivot to the ground pivot. The coupler link connects the two moving pivots, joints $\mathrm{b}$ and $\mathrm{c}$, thereby coupling the input link to the output link [22]. The couple link can trace out the path trajectory when driving the input link. The four-bar linkage is a simple mechanism. It is very versatile and is used in thousands of applications such as level luffing crane, automobile hood linkage, and drive linkage for a lawn sprinkler [22]. The four-bar linkage is selected as the basic link set to drive the RGB lamps. Based on Grashof's Law, to drive a four-bar linkage, it is assumed that



FIgURE 3: Four-bar mechanism.

the length of the shortest link is $r_{s}$ and that of the longest is $r_{l}$, while those of the other two are $r_{p}$ and $r_{q}$, if the link lengths satisfy the following:

$$
r_{s}+r_{l} \leqq r_{p}+r_{q}
$$

A four-bar mechanism that meets Grashof's Law is developed using (1), as shown in Figure 4, which is a 3D simulation of double-crank link mechanism. Therefore, this study used three four-bar mechanisms to carry red, green, and blue LEDs, respectively. The mechanism of color mixing is shown as Figure 4(a). Firstly, the RGB LEDs are set on coupler links of three four-bar linkages, respectively. By driving the input link of linkages, the couple links which install the RGB LEDs will produce the relative motion. And then this mechanism can achieve the color mixing image gradually. Figure 4(b) shows the path trajectory of RGB lighting.

Figure 4 shows that the four-bar mechanism is in fact a double-crank linkage where the four bars include double crank links used for input and output, a couple link with a tilting angle, and a fixed link, as shown in Figure 5. The light mixing mechanism design mentioned in this study consists of 3 double-crank mechanisms, and each of the RGB LEDs' lamp-type is installed on the couple links of the three fourbar mechanisms. The couple link has a tilting angle that is designed to produce the effects of focusing and mixing the RGB colors as the mechanisms operate, thus achieving the function of scenario illumination.

2.2. Experiment Model. The tilting angle of couple link in the mechanism is used for light mixing. Therefore, the parameter of the tilting angle is obtained by maximizing the overlapping area of the RGB lights. In Figure 6, the black part is where all the three colors overlap. The couple links on which the RGB LEDs are installed tilt from 10 to 70 degrees. The simulation tool, SolidWorks motion, is used to determine the optimized tilting angle for the couple links. As the mechanism operates, it drives the RGB lights to project on a plane to create light trajectories from the area of the part where the three basic colors overlap. The overlapping areas for each tilting angle are shown in Figure 7. The result from the simulation of SolidWorks suggests that the overlapping of color lights is achieved with the tilting angle of LED-equipped couple links ranging from 25 to 65 degrees as the mechanism operates; that is, the effects of focusing and overlapping the RGB lights 



(a) Three four-bar mechanisms



(b) RGB lighting path

FIGURE 4: 3D simulation of color mixing mechanism.

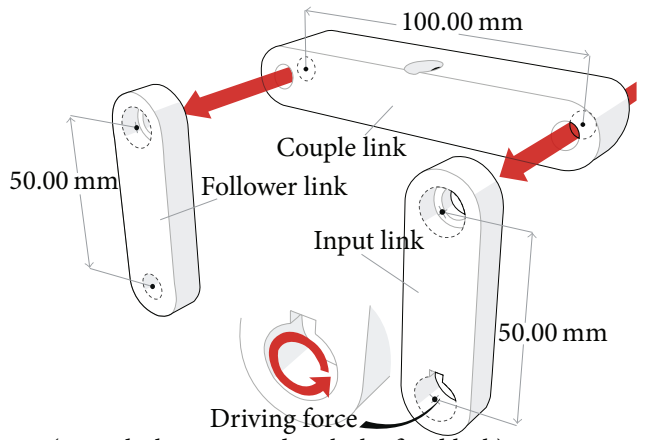

(input link connected with the fixed link)

FIGURE 5: Double-crank four-bar mechanism.



FIGURE 6: Top view of the RGB color trajectory overlapping.

are produced. It is also found that when the couple link tilts at 40 degrees, the RGB color lights project on the plane and create an overlap with the optimized area, thus achieving local light mixing. Therefore, the couple link in this mechanism is designed to tilt at 40 degrees for RGB light mixing, as shown in Figure 8. As a result, three four-bar mechanisms, each consisting of a couple link tilting at 40 degrees, double-crank links for input and output, and a fixed link, are combined into a RGB LED light mixing mechanism. The optical simulation program, LightTools, is brought in as the design tool for light mixing and to simulate the mixing and configuration of the mixing mechanism.

Most of the RGB color mixing is using the control system to produce the color of lights. The concept of this design is applying the mechanism motion approach to achieve the color of lights. This light mixing mechanism consists of 3 sets of double-crank four-bar mechanisms. The synchronized

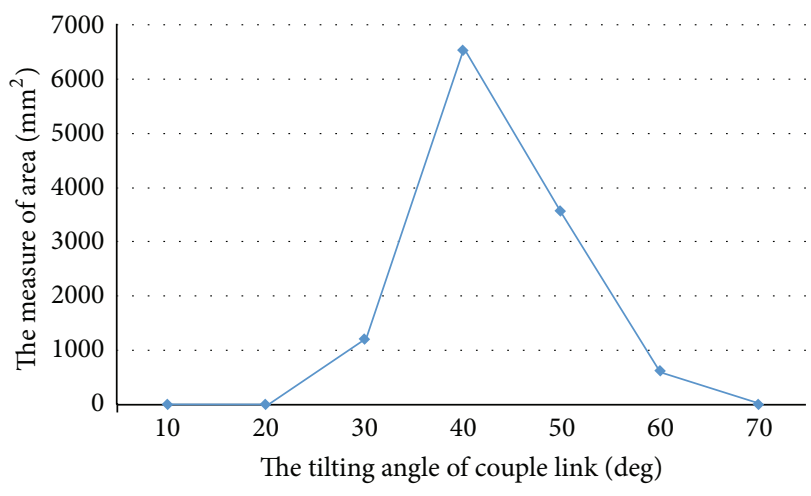

FIGURE 7: RGB light overlapping area.

movements of the mechanism generate the mixing effects desired. It works by connecting a motor on the fixed link of each linkage to drive the input and output cranks for circular rotation movement at constant speed. At the same time, the couple link which connected with the input and output link will also produce the relative motion. In addition, the 3 linkage sets are placed on three sides of a hexagon to allow crossing of light trajectories as the mechanism rotates. The color presentation from the mixing changes constantly due to the continuous rotations of cranks. As the angle of the couple link is confirmed, a mockup is produce to verify the feasibility of light mixing design. First, the RGB light mixing mechanism requires confirmation of its stability, manufacturability, and movement reasonability to allow the ambience light mixing which can match with the manufacturing practice. Computer simulation is conducted to simulate the mechanism movement and shop drawings of part manufacturing are printed. Aluminum alloy and acrylic plastics are selected as the primary materials and parts are machined using CNC. For final assembly, the RGB LEDs are embedded into the mechanism and connected to battery for power to simulate the feasibility of light mixing mechanism. Figure 9 is the 3D simulation of the mockup. This RGB light mixing mechanism consists of five parts: the double-crank linkage design, bearing socket set design, geared powers source design, transmission shaft design, and support design.

Double-Crank Linkage Design. The mechanism proposed in this study is provided with a ball bearing assembled with nuts 

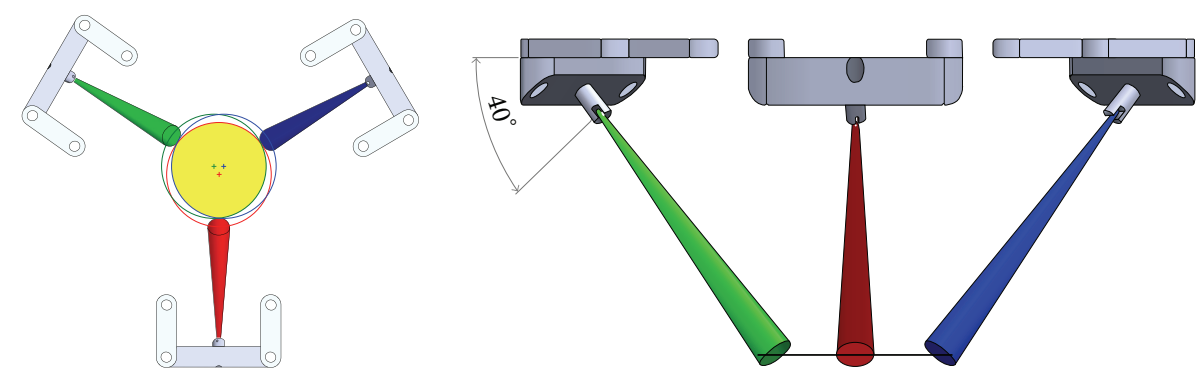

FIGURE 8: Simulation of projection plane with the couple link tilted at 40 degrees.

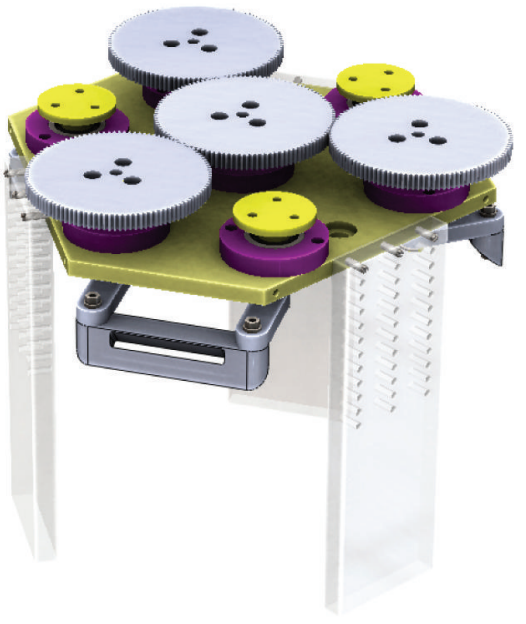

FIGURE 9: 3D simulation of the actual RGB mixing mechanism.

and bolts in order to increase the overall stability and strength of the mechanism. The ball bearing is as the revolute joints. This ball bearing is added for the improvement of stability and smoothness of movements between the links, while the nuts and bolts are assembled to secure the connection between the double-crank link and couple link, as shown in Figure 10.

Bearing Socket Set Design. The bearing socket is designed to be secured on the hexagonal base plate with bolts. It is where the two ball bearings are place, one on the top and the other at the bottom, to ensure that the transmission shaft rotates smoothly to drive the four-bar mechanism and improve the stability of mechanism when driving them. Aluminum alloy is selected for better strength and accuracy, as shown in Figure 11.

Geared Powers Source Design. The diameter of gear is determined to be $111.18 \mathrm{~mm}$ using the gear design equation. With module being equal to 1, pressure angle is equal to 20 degrees and 111 teeth as the machining parameters; the middle gear is produced as the power source to drive the other 3 gears. The gears have to be fastened on the transmission shaft with 3 bolts, as shown in Figure 12.

Transmission Shaft Design. The transmission shaft is the core of the mechanism that transmits driving power. It joins with a fixed gear to become a single component and drives the fourbar mechanism below it. Therefore, a set screw is fastened at the bottom end of the shaft through the link, as shown in Figure 13.

Support Design. The support means the fixed link of the proposed mechanism. The support is designed to allow the adjustment of distance to the RGB projection plane. Therefore, acrylic is used and holes are drilled in the support to allow height adjustment, as shown in Figure 14. The acrylic provides visibility that allows observation during the verification of RGB light mixing. Also, holes are drilled in the sides of the hexagon to allow movement of acrylic support and better mobility.

\section{Results and Discussions}

A RGB light mixing mechanism is proposed which generates light mixing effect unlike typical control techniques. A fourbar mechanism is introduced as the carrier. Each of the RGB LEDs lamp-type is installed on a couple link tilted at an angle. The cranks used generate relative movements in other links to produce the light focusing and mixing effects as the red, green, and blue lights are projected on a plane. Optical simulation is first introduced to verify the light mixing effect before a mockup is produced to verify the feasibility of light mixing technique proposed.

3.1. The Results of Optical Simulation. In the optical simulation, the distribution curve of luminous intensity in the light source conditions of the RGB lamps set up on the couple link was measured from the actual LED lamps, as shown in Figure 15. The wavelengths of the RGB LEDs lamp-type were measured as well, as shown in Figure 16. For lighting conditions, the light lumen or the light flux is measurement through the actual RGB lamps. The irradiation angle is 110 degrees by measuring the actual RGB lamps. The projection distance from light source is set at $80 \mathrm{~mm}$ to $120 \mathrm{~mm}$. And the receiver plane is 1000 square millimeters. For this projection distance, the irradiation angle is 110 degrees. Thus, it will guarantee the receiver plane can receive the mixed color. Furthermore, the irradiation angle will become narrow if the projection distance is raised. Because the cranks of the mechanism operated from 0 to 360 degrees, 120 sets of color mixing simulation results were obtained for CIE color mixing 


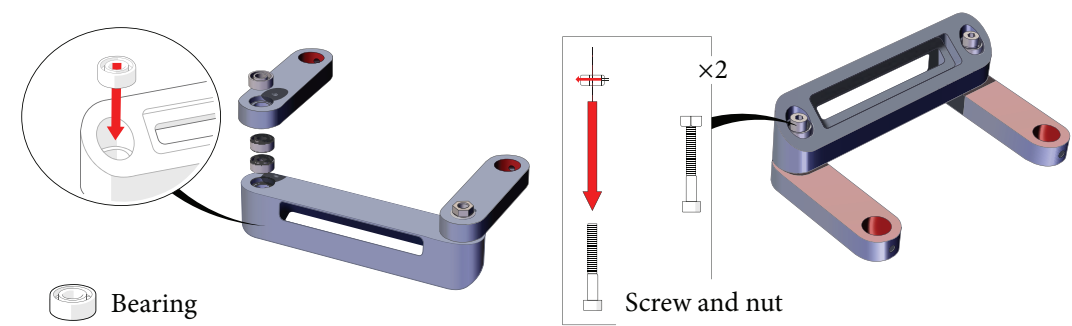

Figure 10: Double-crank linkage design.
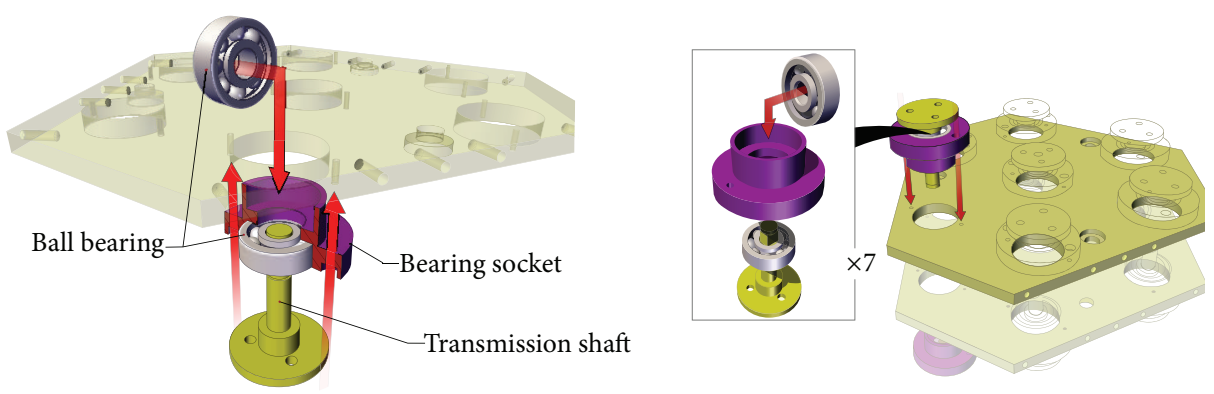

FIGURE 11: Bearing socket set design.

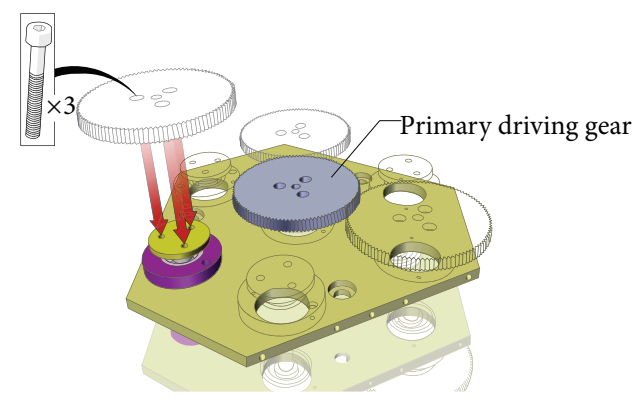

FIGURE 12: Geared powers source design.

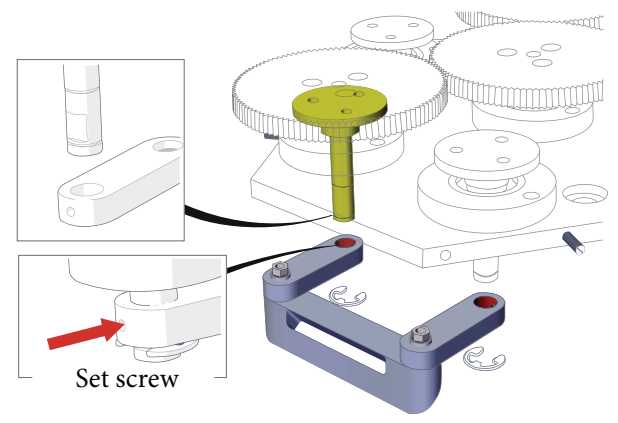

FIgURE 13: Transmission shaft design.

measurements at every 3 degrees of the crank rotation for color mixing simulation.

The RGB color mixing mechanism proposed was converted into the program LightTools based on the relative positions of each of the links in the four-bar mechanism when the cranks rotated every 3 degrees, as to facilitate the RGB color mixing simulation. As a result, 120 sets of color mixing simulation results were obtained as the cranks rotated 360 degrees. The 120 sets of RGB Raster Chart reveal that the color mixing effect was a continuous and dynamic change with 


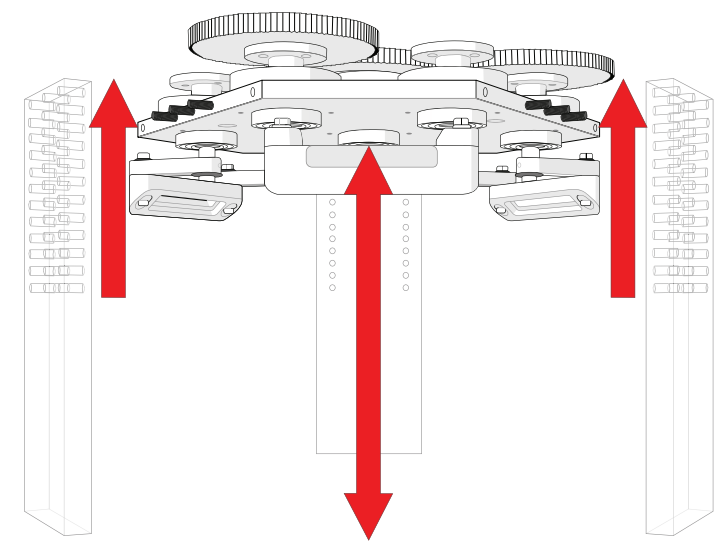

Figure 14: Support design.

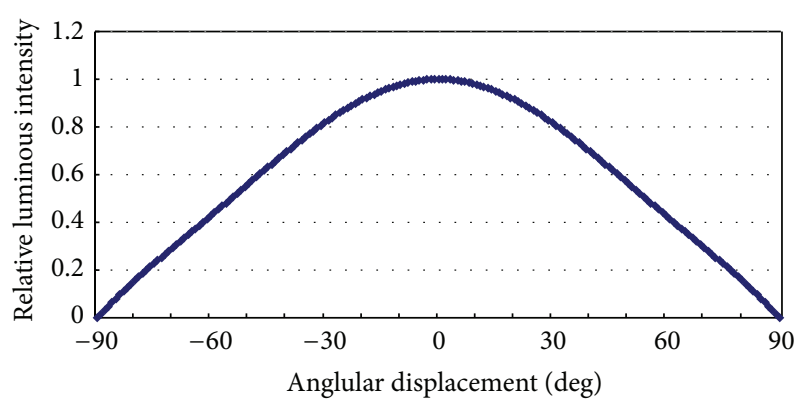

FIGURE 15: Radiation patterns.

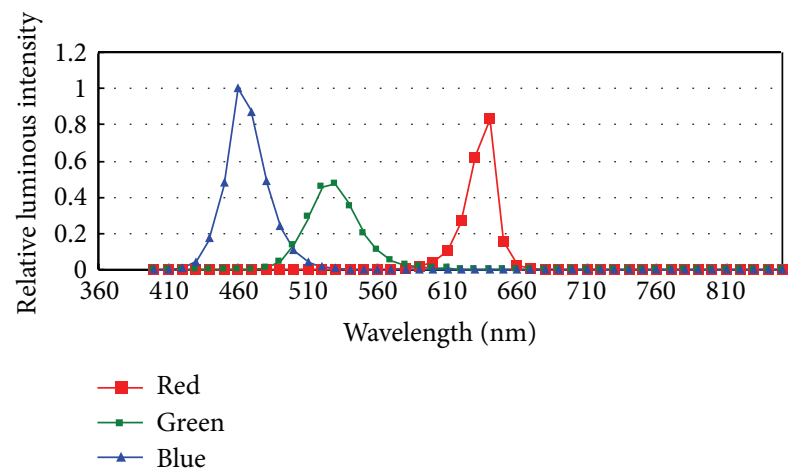

FIgURE 16: The luminous spectra of RGB LEDs.

RGB colors driven by the mechanism. The projection on the surface provided images having multiple color mixing effects, as shown in Figure 17. Figure 17 shows the color mixing effects with the cranks rotating at various angles. Due to the number of color mixing images, only the images at every 30 degrees are presented. After the RGB Raster Charts were obtained, this study was able to learn from the color mixing simulation the chromaticity color coordinates corresponding to the colors of mixing at every 3 degrees of crank rotation, as shown in Figure 18. The chromaticity color coordinates show the color distribution trends of every mixing, and from them it is found that the RGB color mixing can be concentrated on certain color patches or distributed over multiple ones as well. Therefore, a mechanism was used in the study to propel the color mixing patterns. Figure 18 shows that the range of color mixing was widely distributed, that is, presentation of multiple colors on the projection surface. In the future, it is possible to achieve a special color mixing effect with local color distribution by adjusting the inclining angles of couple links and the rotation speed of the cranks in the three mechanisms. Moreover, we will be able to investigate the purity and temperature of colors from the chromaticity color coordinates in order to facilitate the light design and applications of visual ergonomics.

Figure 17 shows that the color of mixing is constantly changing as the crank rotates around. It is found that the color of mixing approaches is white when the cranks rotates between $240^{\circ}$ and $300^{\circ}$, as shown in Figure 19. The mixing result suggests that production of white light is possible with specific crank angles when using this mechanism as the carrier for RGB light mixing. The mechanical light mixing technique with the introduction of mechanism produces the same light mixing effect as that using electronic control does. Figure 20 shows the light mixing effect generated by electronic control in [23], where the outstanding light transmitting characteristics of PMMA plastic are used for the mixing simulation of RGB LEDs. Figures 20(a) and 20(b) are the same simulation results but at difference angles. Therefore, it is possible to generate white light illumination of specific color temperature or light mixing illumination of specific color using this mixing technique with rotating mechanism. A possible application of this optical design is the examination of pathological changes at cellular level in biomedical technology, as different cells emit different lights of different wavelength. A means of lighting with adjustable color temperature is helpful for biomedical engineering tests.

3.2. Verification with Mockup. The parts are machined and assembled into the verification mockup. The mockup is connected to batteries for power and the RGB LEDs are installed on the couple links of the mechanism. The mockup is shown in Figure 21. 


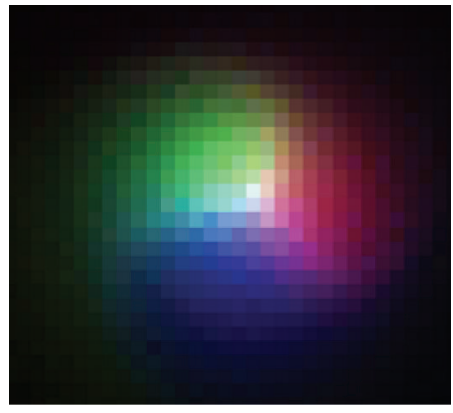

(a) $0^{\circ}$

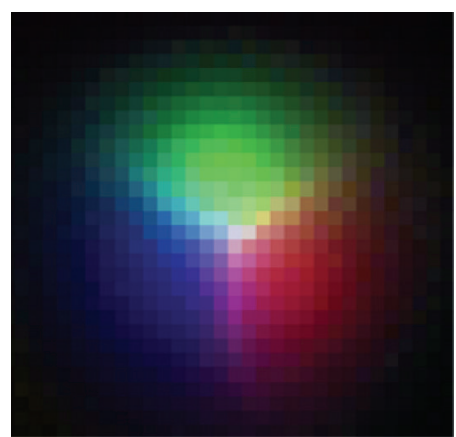

(d) $90^{\circ}$

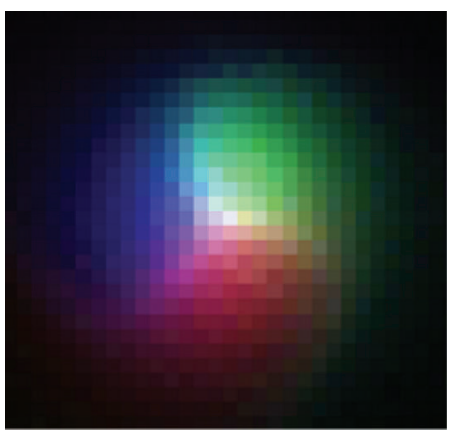

(g) $180^{\circ}$

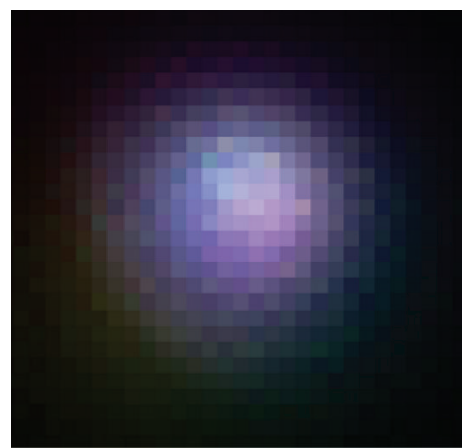

(j) $270^{\circ}$

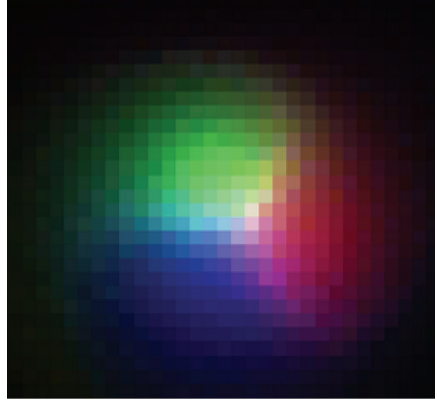

(b) $30^{\circ}$

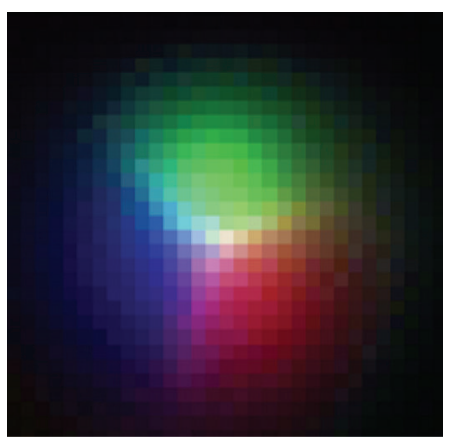

(e) $120^{\circ}$

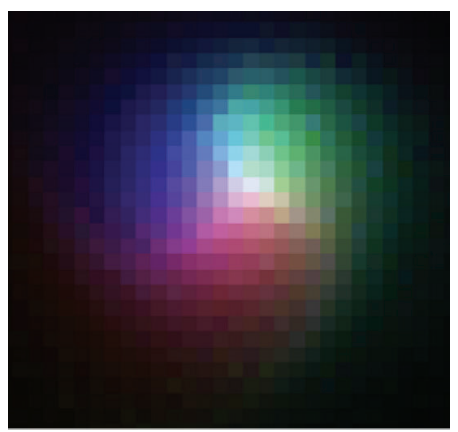

(h) $210^{\circ}$

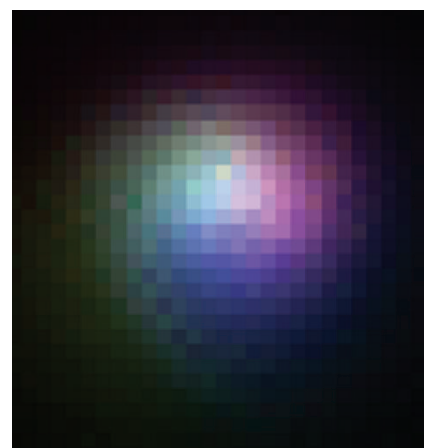

(k) $300^{\circ}$

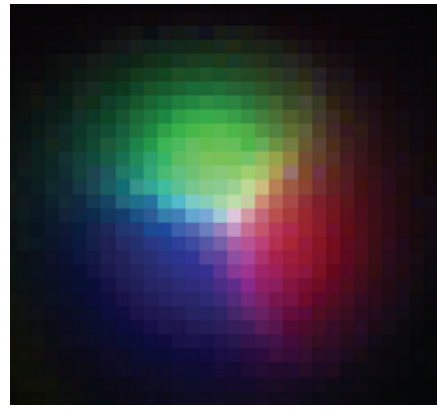

(c) $60^{\circ}$

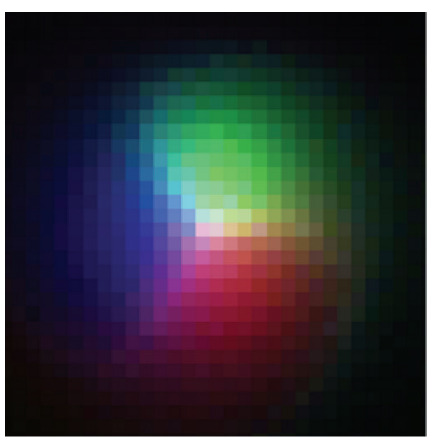

(f) $150^{\circ}$

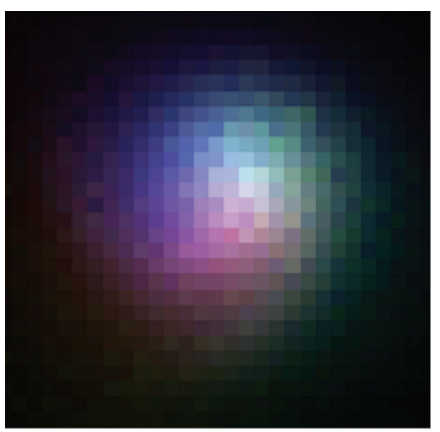

(i) $240^{\circ}$

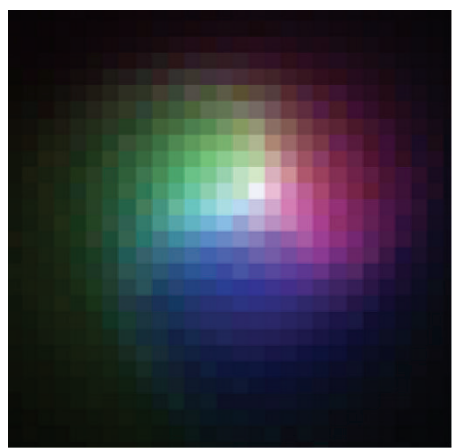

(l) $330^{\circ}$

FIGURE 17: Modeled output color mixing when crank is rotated from $0^{\circ}$ to $360^{\circ}$.

Figure 22 provides the light mixing effect as the mockup mechanism starts to operate. White glows are generated on the projection plane where the RGB lights overlap. This verifies that the RGB light mixing mechanism produces the desired light mixing effect. As the crank rotates continuously, the color of the mixing changes constantly. This is capable of satisfying the changes of colorful scenarios that people love. Hence, for the light mixing simulation using the mechanism proposed in this study as the carrier of RGB LEDs, the results suggest the difference between the light mixing technique 


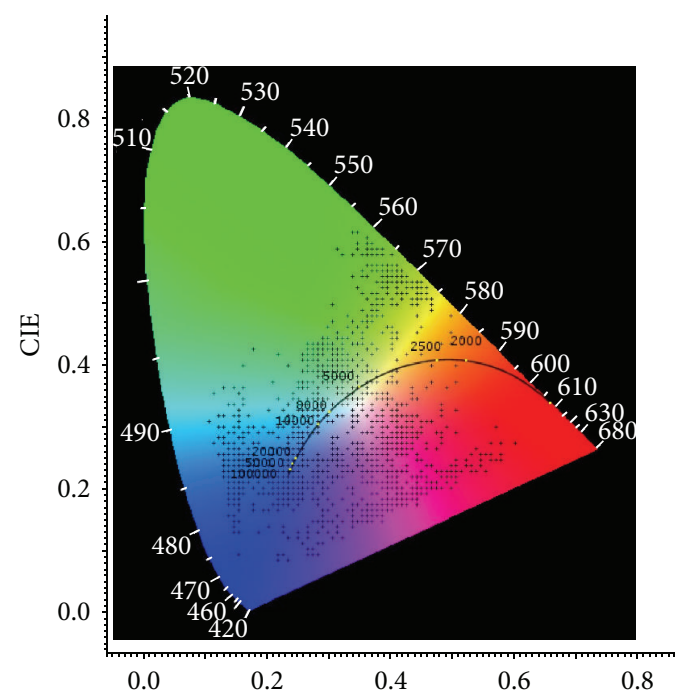

(a) $0^{\circ}$

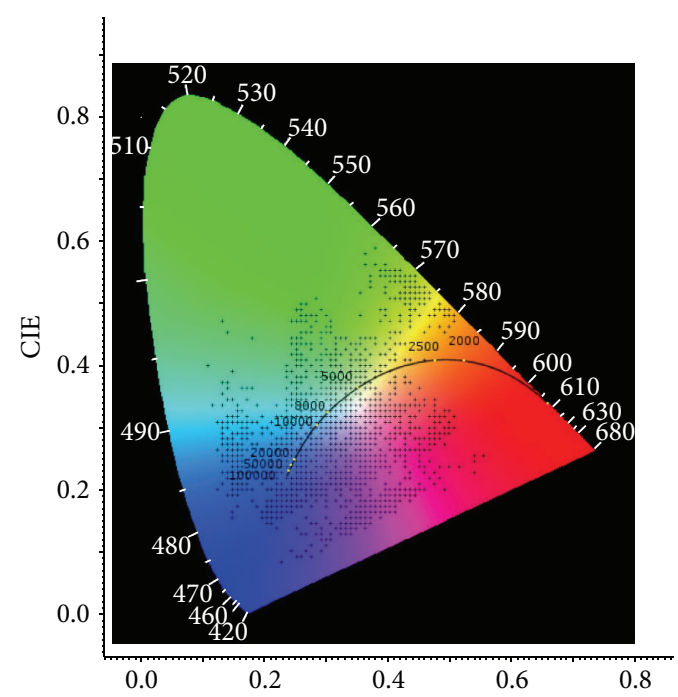

(c) $60^{\circ}$

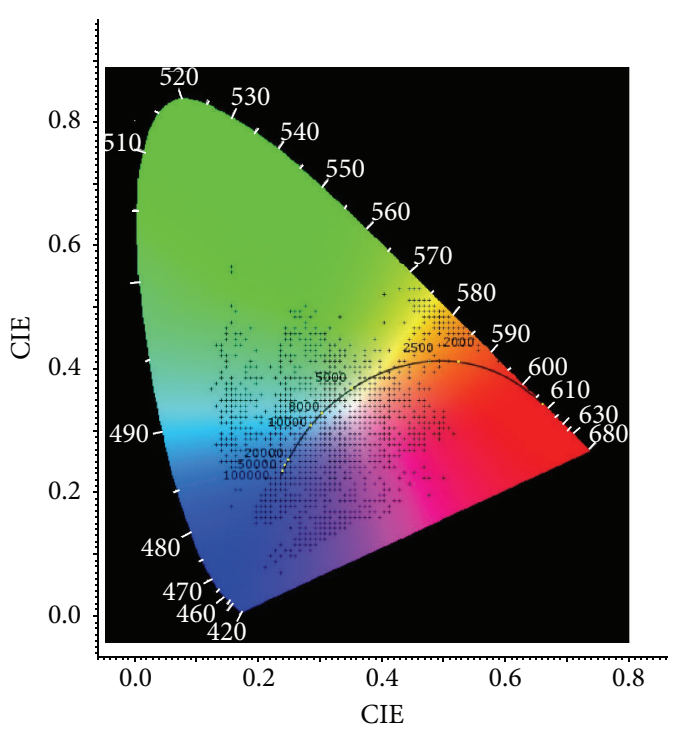

(e) $120^{\circ}$

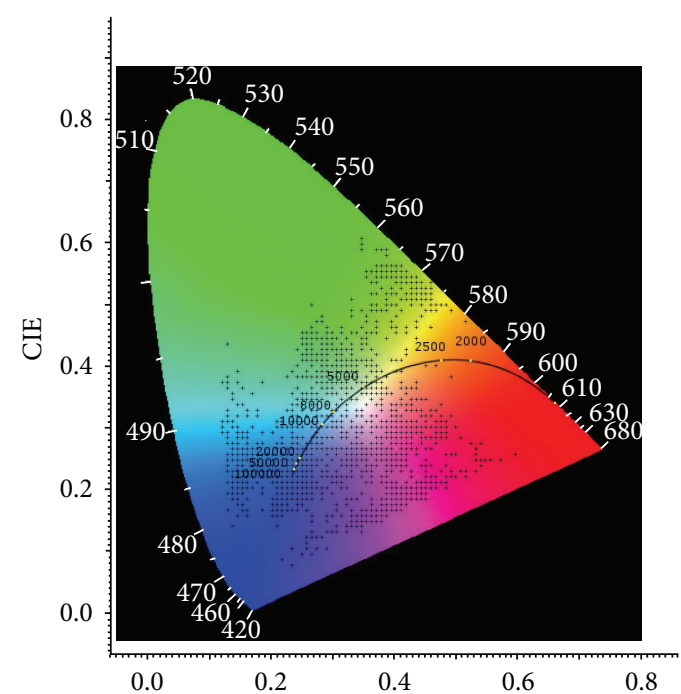

(b) $30^{\circ}$

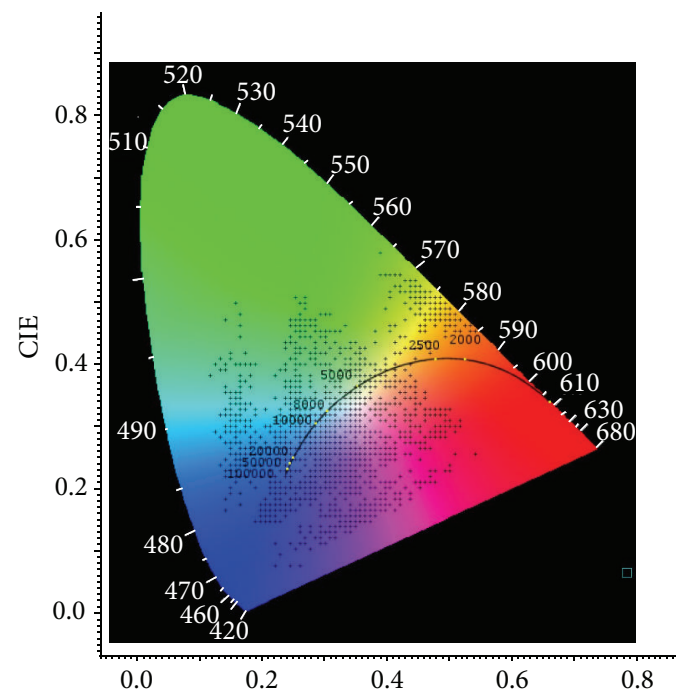

(d) $90^{\circ}$

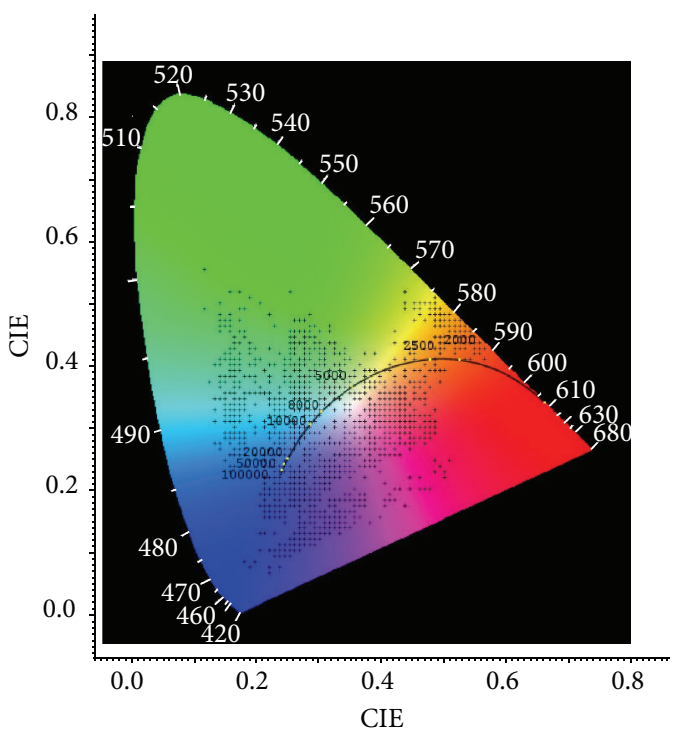

(f) $150^{\circ}$

FIGURE 18: Continued 


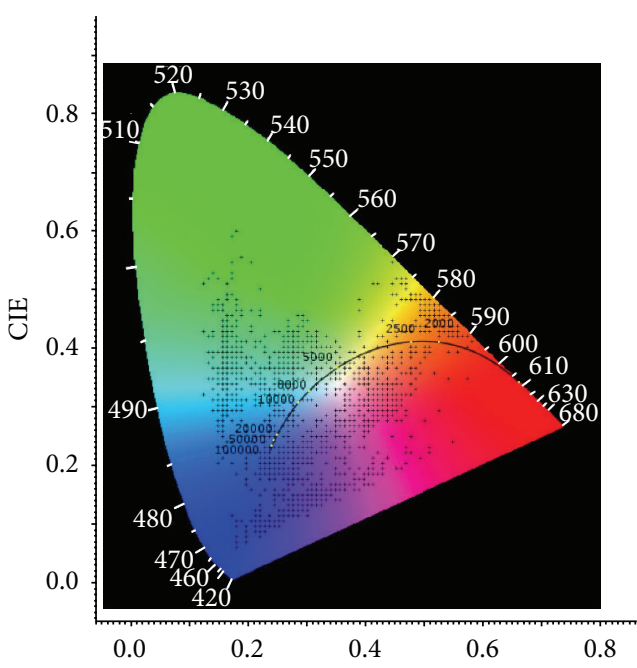

(g) $180^{\circ}$

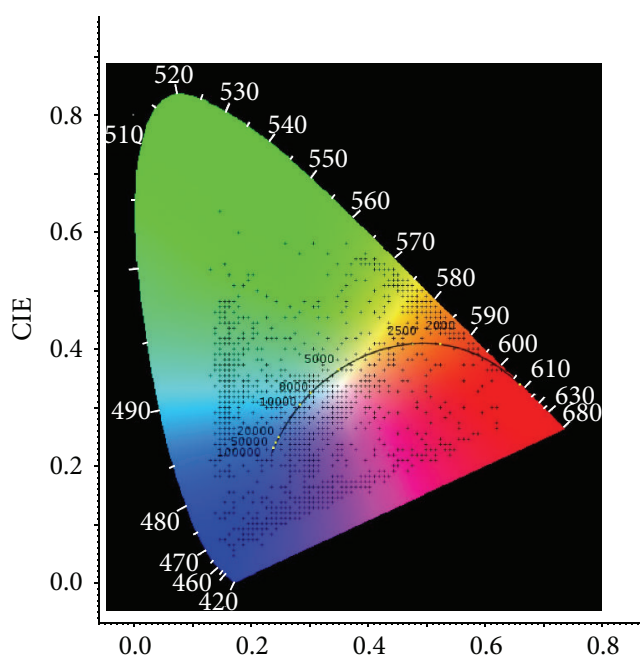

(i) $240^{\circ}$

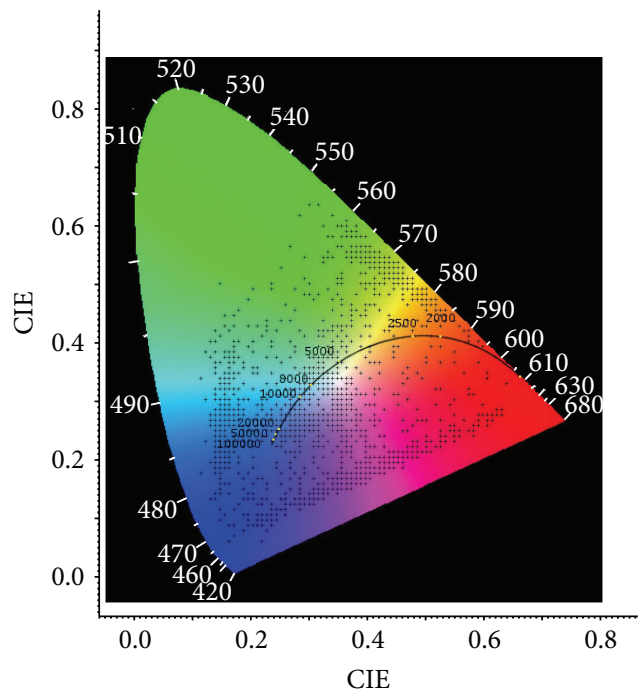

(k) $300^{\circ}$

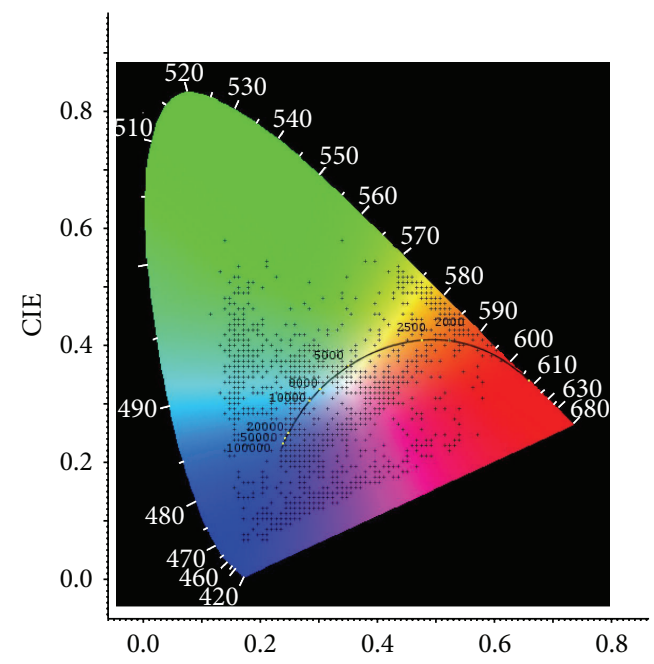

(h) $210^{\circ}$

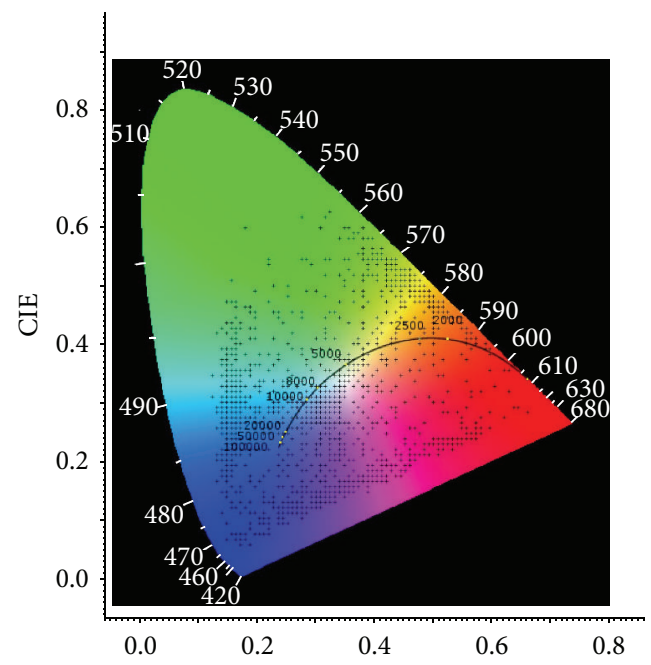

(j) $270^{\circ}$



(l) $330^{\circ}$

FIGURE 18: The chromaticity color coordinates for the color mixing mechanism. 


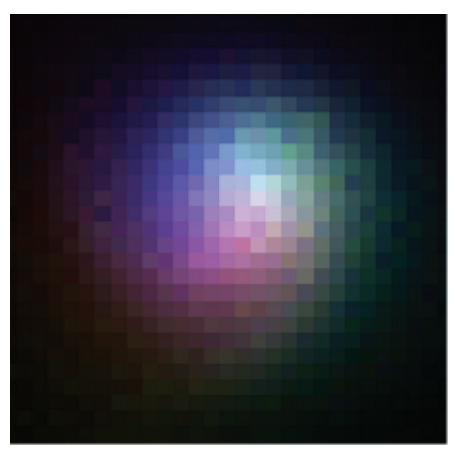

(a) $240^{\circ}$

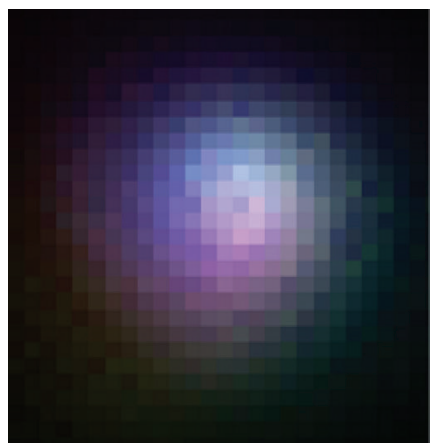

(d) $255^{\circ}$

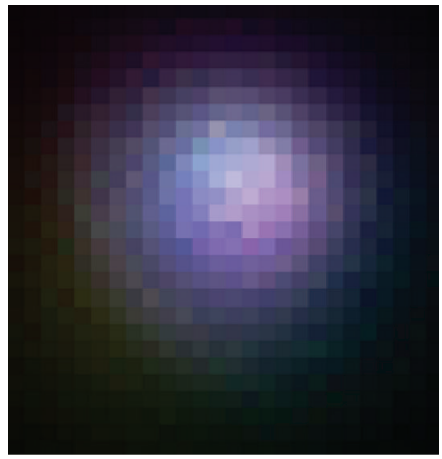

(g) $273^{\circ}$

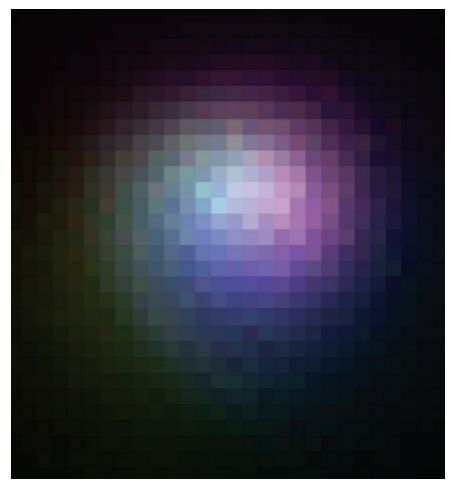

(j) $291^{\circ}$

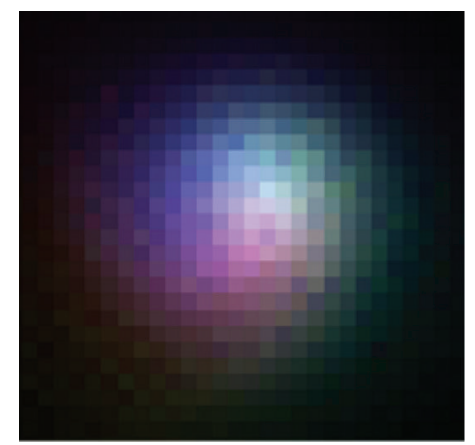

(b) $243^{\circ}$

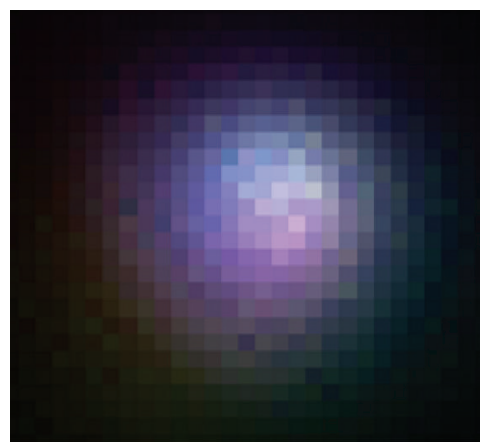

(e) $261^{\circ}$

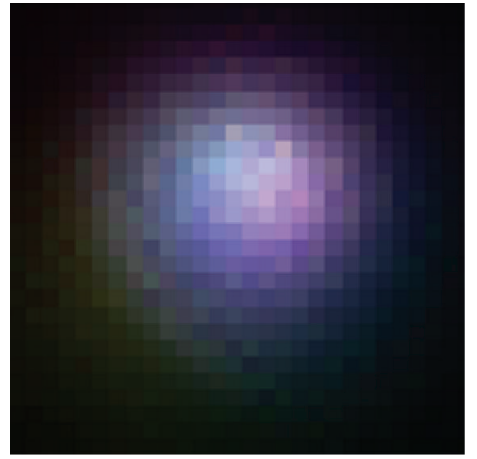

(h) $279^{\circ}$

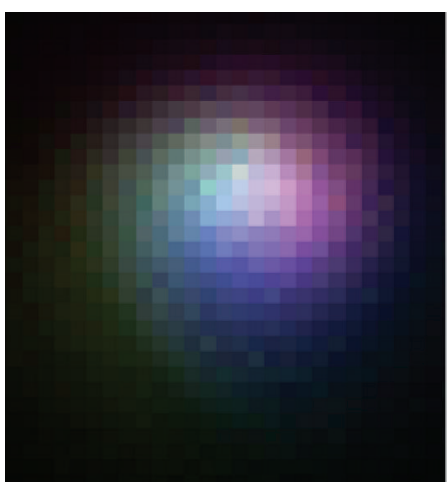

(k) $294^{\circ}$

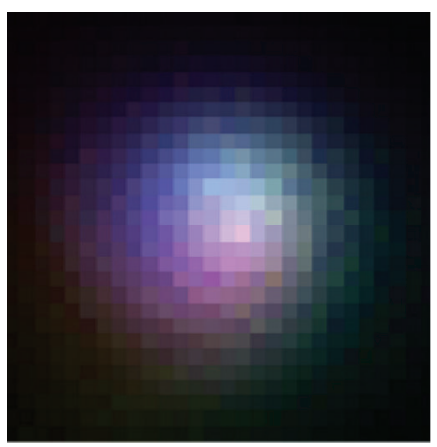

(c) $249^{\circ}$

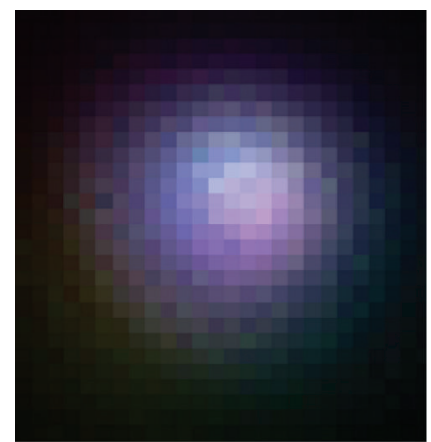

(f) $267^{\circ}$

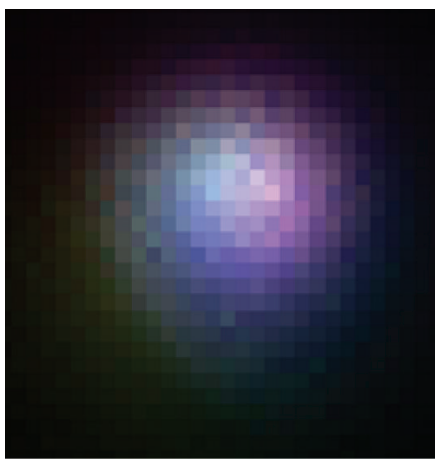

(i) $285^{\circ}$

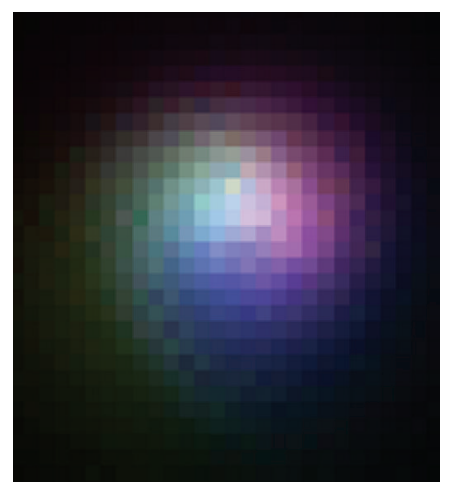

(l) $300^{\circ}$

FIGURE 19: RGB LEDs can be combined to produce white light. 


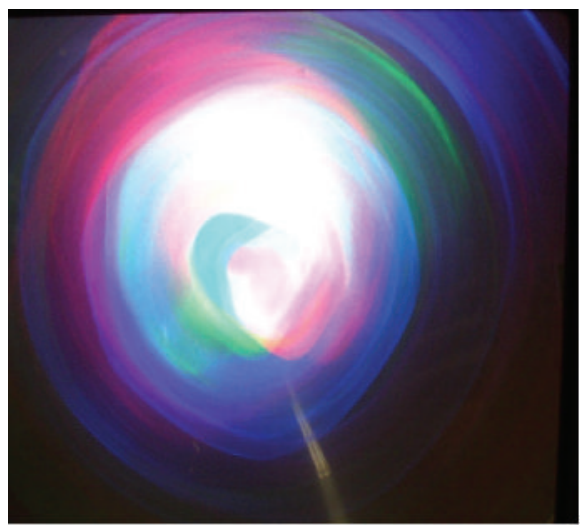

(a)

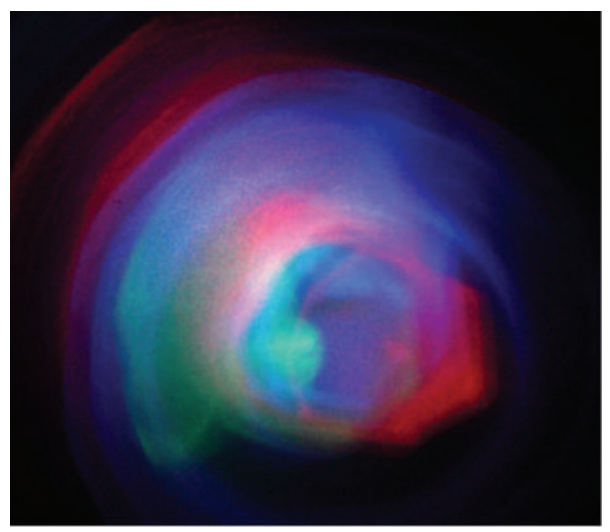

(b)

Figure 20: Photographs of experimental results [23].

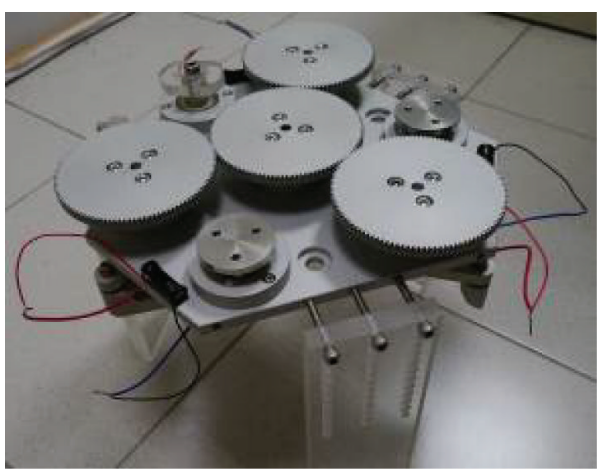

(a) Mockup assembly

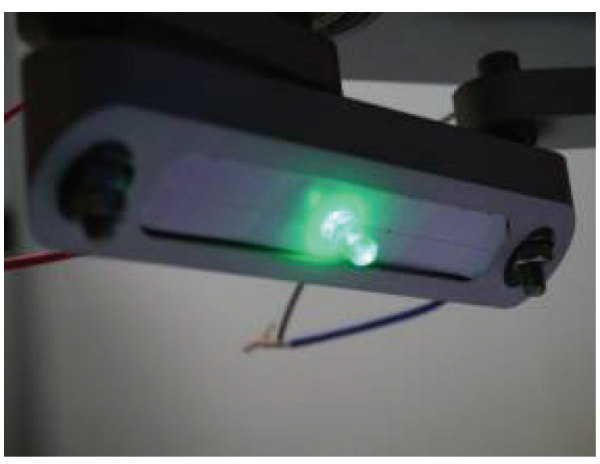

(c) Green LED

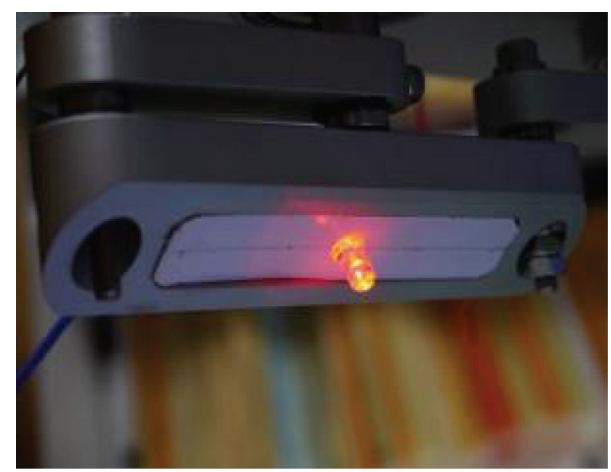

(b) Red LED

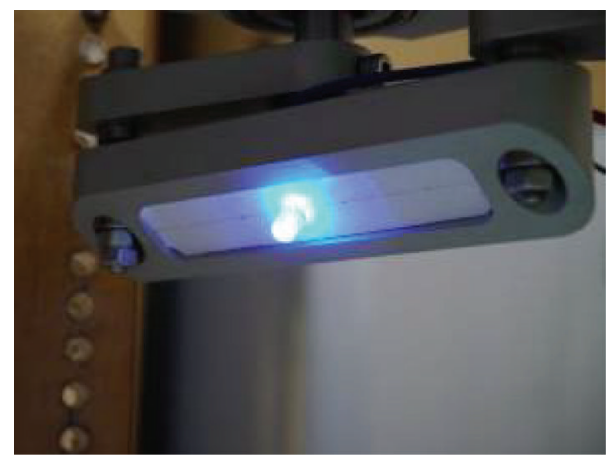

(d) Blue LED

Figure 21: Mockup.

driven by a mechanism and that by electronic control, as shown in Table 1.

\section{Conclusions}

An innovative light mixing technique is proposed. A mechanism that mixes red, green, and blue lights is designed based on applicable mechanism design principles. Each of the RGB LEDs' lamp-type is installed on one of the three four-bar mechanisms. The couple link tilted at an angle works with the cranks to drive the projection of RGB lights on a plane for mixing. The optical simulation conducted and mockup produced verify that the light mixing technique with the mechanism as carrier produces an extensive range of mixing projection and the color of mixing varies widely. Unlike the mixing techniques with electronic control, this design provides multiple choices for lighting fixture designers who are allowed to provide visibility and comfort to human 


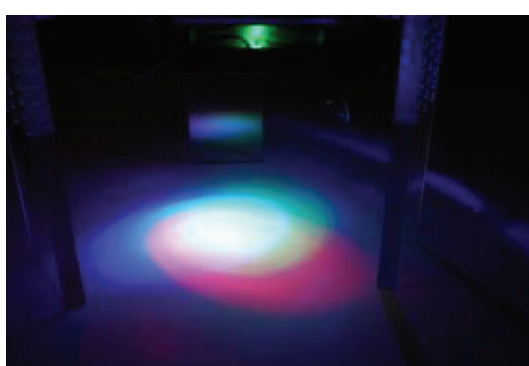

(a)

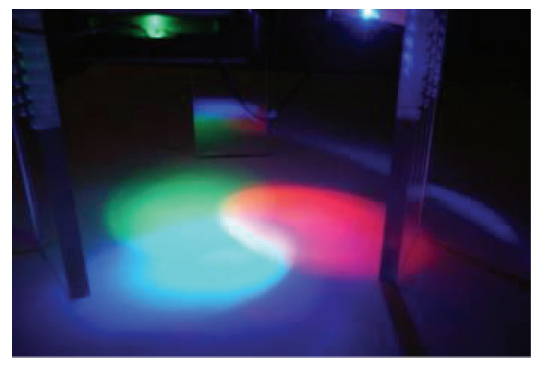

(d)

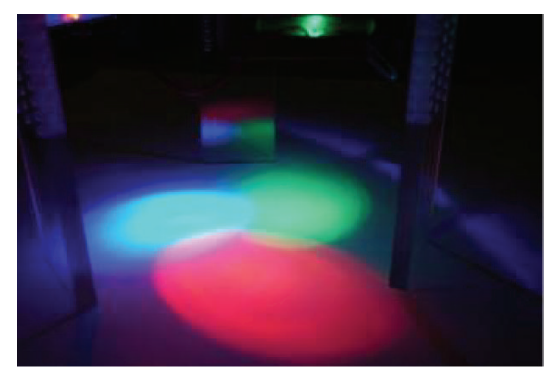

(b)

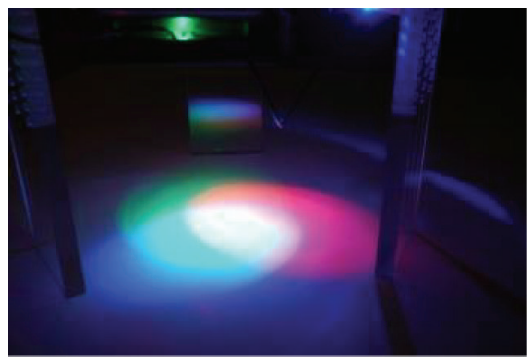

(e)

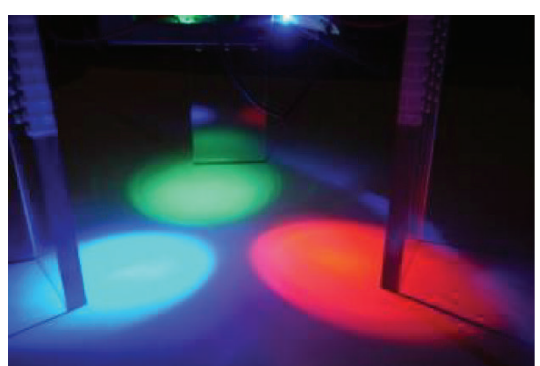

(c)

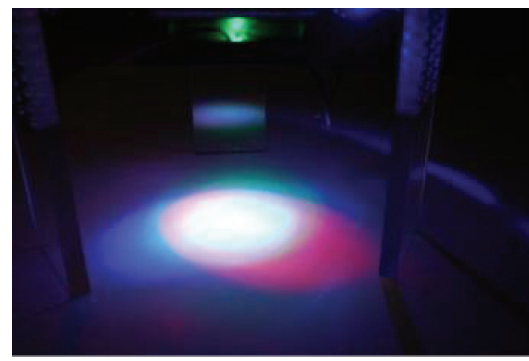

(f)

FIGURE 22: Light mixing effect produced by the mockup.

TABLE 1: Comparison of color mixing techniques.

\begin{tabular}{ll}
\hline Mechanism-driven color mixing & Electronically controlled color mixing \\
\hline $\begin{array}{l}\text { A four-bar mechanism is used as the carrier; RGB LEDs are } \\
\text { mounted on the couple links of the mechanism; the rotation of } \\
\text { cranks is used for color mixing simulation. }\end{array}$ & $\begin{array}{l}\text { Inputs of voltage and current are used to project light on different } \\
\text { lenses, and the color mixing effect can be enhanced with reflection } \\
\text { and refraction. Multiple lenses are required to achieve color mixing } \\
\text { effect. }\end{array}$ \\
\hline
\end{tabular}

The rotation angle of cranks and the inclining angle of couple links are adjusted to perform mixing of the three colors of RGB.

The quantity of voltage and current inputted is used to control how much the RGB colors are mixed.

The colors projected on the same surface have direct single colors and area of multiple gradual colors.

There can be a large number of color changes, but at the end, the colors projected on the surface are single colors.

The range of lighting is wide; that is, the color mixing can be projected over a large area, as the cranks rotate $360^{\circ}$ in the mechanism.

The lighting range is limited to a certain degree.

Due to wide range of lighting, the combination with LED lights of greater lumens may increase the projection distance. The mechanism style color mixing is that the LEDs of three different colors are mounted on the mechanism independently and create mixing that is independent of one another. This allows the users to adjust the lighting directions of the three mechanisms to achieve mixing of specific colors.

As the three mechanisms rotate at the same time, the entire color mixing mechanism is large in size and requires large space for the operation.

Electronic color mixing works by packaging the LEDs of three colors on one chip. Due to this limit, the light color may not be easily focused as the projection distance increases, thus the light dispersion.

The electronically controlled color mixing devices are smaller in size.

eyes with the illumination source based on optical element design, color implication, visual ergonomics, and usability engineering assessment. It is possible to introduce a lighting fixture featuring this mechanism on large stages, pubs, karaoke, and other places that need to create the ambience with special multicolor light source. This allows the creation of environment with specific ambience and scenario but also saves power bills and achieves energy saving and $\mathrm{CO}_{2}$ reduction. The study result suggests that the color of mixing approaches is white as the cranks in each set of mechanism rotate between $240^{\circ}$ and $300^{\circ}$ as all three mechanisms operate synchronously. The RGB lights are projected independently. Therefore, light sources of specific color temperature can be produced by manipulating the rotation angles of crank and the tilting angles of couple link and thus used for tests of biomedical engineering or medical cosmetology. 


\section{Conflict of Interests}

The authors declare that there is no conflict of interests regarding the publication of this paper.

\section{Acknowledgment}

Funding support for this project, MOST 103-2221-E-366001 and MOST 103-2632-E-366-001, was received from the Ministry of Science and Technology (Taiwan).

\section{References}

[1] M. Lee and G. Cho, "Measurement of human sensation for developing sensible textiles," Human Factors and Ergonomics In Manufacturing, vol. 19, no. 2, pp. 168-176, 2009.

[2] Shin-Chi Lights, http://www.shin-chi.tw.

[3] H. C. Chen, G. Y. Wu, C. H. Yang, P. Y. Chen, M. J. Lai, and K. T. Huang, "Using artificial neural networks approach for the color enhance of high power LEDs," in 12th International Conference on Solid State Lighting and 4th International Conference on White LEDs and Solid State Lighting, vol. 8484 of Proceedings of SPIE, p. 84841G, San Diego, Calif, USA, October 2012.

[4] H. Ou, D. Corell, Y. Ou, P. B. Poulsen, C. Dam-Hansen, and P.-M. Petersen, "Spectral design flexibility of LED brings better life," in Light-Emitting Diodes: Materials, Devices, and Applications for Solid State Lighting XVI, vol. 8278 of Proceedings of SPIE, January 2012.

[5] C.-C. Sun, I. Moreno, Y.-C. Lo, B.-C. Chiu, and W.-T. Chien, "Collimating lamp with well color mixing of red/green/blue LEDs," Optics Express, vol. 20, no. 1, pp. A75-A84, 2012.

[6] I. Moreno, N. Rodriguez, and J. C. Basilio, "Simultaneous color-mixing and collimation within LED package," in Current Developments in Lens Design and Optical Engineering XIV, vol. 8841 of Proceedings of SPIE, pp. 884102-1-827802-5, 2013.

[7] D. A. Steigerwald, J. C. Bhat, D. Collins et al., "Illumination with solid state lighting technology," IEEE Journal on Selected Topics in Quantum Electronics, vol. 8, no. 2, pp. 310-320, 2002.

[8] Y. Ohno, "Spectral design considerations for white LED color rendering," Optical Engineering, vol. 44, no. 11, Article ID 111302, 2005.

[9] J. H. Oh, J. R. Oh, H. K. Park, Y. Sung, and Y. R. Do, “New paradigm of multi-chip white LEDs: combination of an InGaN blue LED and full down-converted phosphor-converted LEDs," Optics Express, vol. 19, pp. A270-A279, 2011.

[10] T. Kari, J. Gadegaard, D. T. Jørgensen, T. Søndergaard, T. G. Pedersen, and K. Pedersen, "Compact lens with circular spot profile for square die LEDs in multi-LED projectors," Applied Optics, vol. 50, no. 24, pp. 4860-4867, 2011.

[11] H. C. Chen, G. Y. Wu, and J. Y. Lin, "Effects of color temperature and luminous efficiency for RGB LEDs mixing with tuning voltage," in 10th International Conference on Solid State Lighting, vol. 7784 of Proceedings of SPIE, pp. 778418-1-778418-8, San Diego, Calif, USA, August 2010.

[12] P. Liu, H. Wang, R. Wu et al., "Uniform illumination design by configuration of LEDs and optimization of LED lens for largescale color-mixing applications," Applied Optics, vol. 52, no. 17, pp. 3998-4005, 2013.

[13] K. Hashimoto, T. Yano, M. Shimizu, and Y. Nayatani, "New method for specifying color-rendering properties of light sources based on feeling of contrast," Color Research and Application, vol. 32, no. 5, pp. 361-371, 2007.
[14] A. Žukauskas, R. Vaicekauskas, and M. Shur, "Solid-state lamps with optimized color saturation ability," Optics Express, vol. 18, no. 3, pp. 2287-2295, 2010.

[15] C.-H. Lee, "Angularly positioned LED-based spatialtemporal color separation system," Optics Express, vol. 20, no. 17, pp. 19109-19118, 2012.

[16] D. Grabovičkić, P. Benítez, and J. C. Miñano, "TIR RXI collimator," Optics Express, vol. 20, no. 1, pp. A51-A61, 2012.

[17] C.-C. Hsieh, Y.-H. Li, and C.-C. Hung, "Modular design of the LED vehicle projector headlamp system," Applied Optics, vol. 52, no. 21, pp. 5221-5229, 2013.

[18] J.-T. Dong, R.-S. Lu, Y.-Q. Shi, R.-X. Xia, Q. Li, and Y. Xu, "Optical design of color light-emitting diode ring light for machine vision inspection," Optical Engineering, vol. 50, no. 4, Article ID 043001, 2011.

[19] C. H. Wang and T. P. Sun, "A novel color mixing control system for light-emitting diodes," in Electro-Optical Remote Sensing, Photonic Technologies, and Applications III, vol. 7482, pp. 74820K-1-74820K-10, 2009.

[20] E. Chen, F. Yu, and T. Guo, "Design of off-axis arranged lightemitting diodes and dual dichroic mirrors based color mixing system for micro-projection display," Applied Optics, vol. 53, no. 6, pp. 1151-1158, 2014.

[21] T. Bonenberger, J. Baumgart, S. Wendel, and C. Neumann, "LED color mixing with diffractive structures," in Light-Emitting Diodes: Materials, Devices, and Applications for Solid State Lighting XVII, vol. 8641 of Proceedings of SPIE, pp. 864107-1864107-12, San Francisco, Calif, USA, March 2013.

[22] A. G. Erdman, G. N. Sandor, and S. Kota, Mechamism Design Analysis and Synthesis, Prentice Hall, Upper Saddle River, NJ, USA, 2001.

[23] C. Deller, G. Smith, and J. Franklin, "Colour mixing LEDs with short microsphere doped acrylic rods," Optics Express, vol. 12, no. 15, pp. 3327-3333, 2004. 

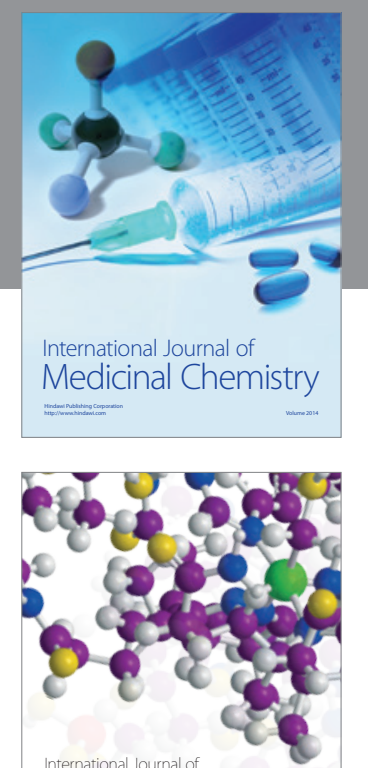

\section{Carbohydrate} Chemistry

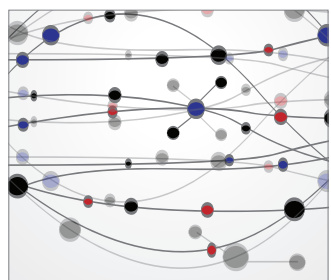

The Scientific World Journal
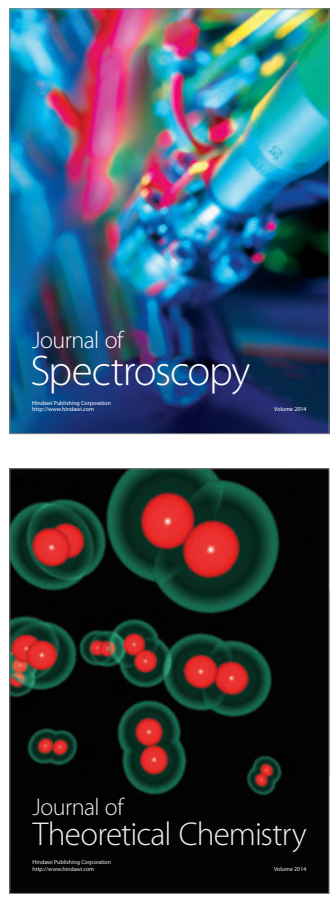
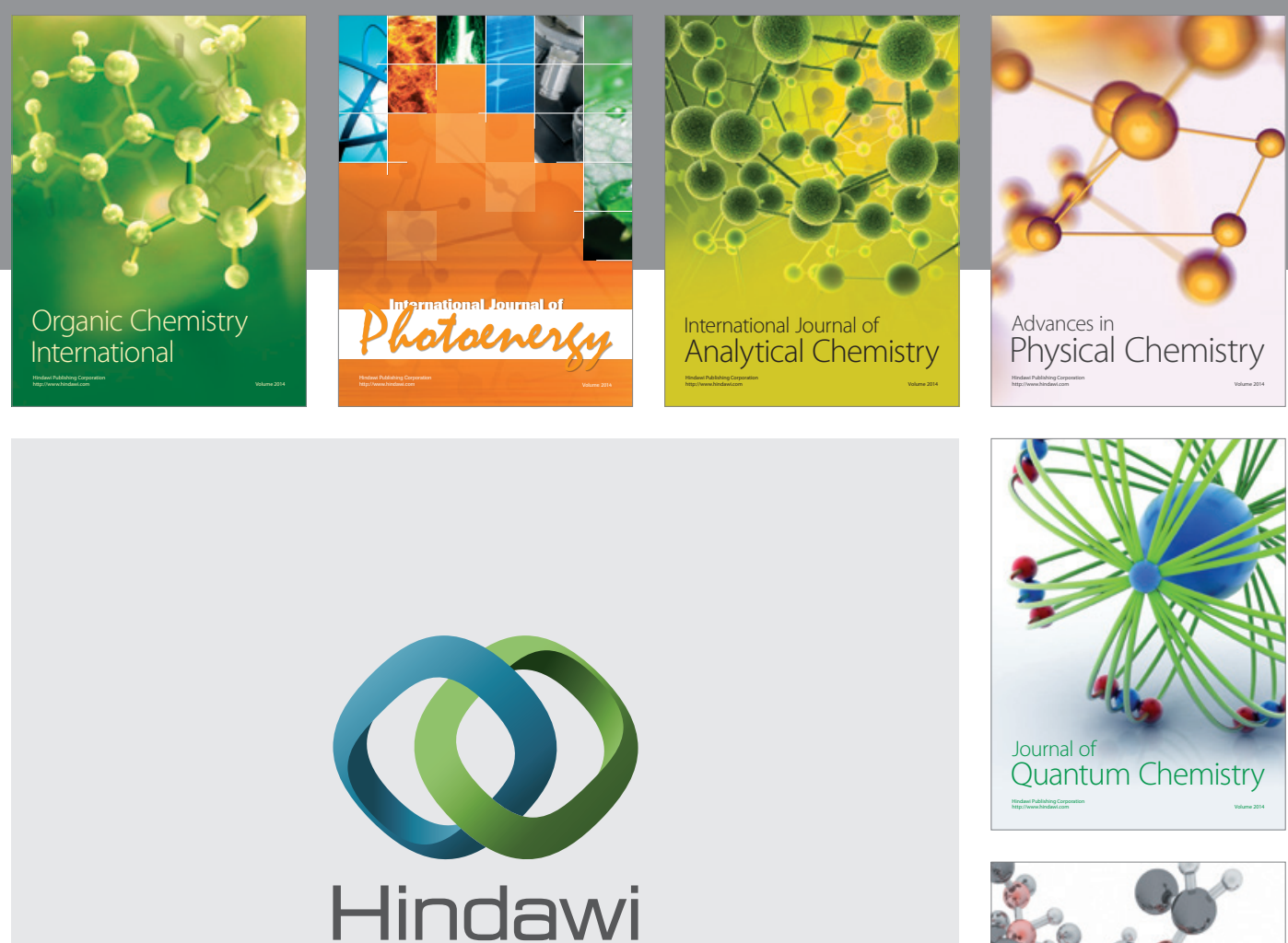

Submit your manuscripts at

http://www.hindawi.com

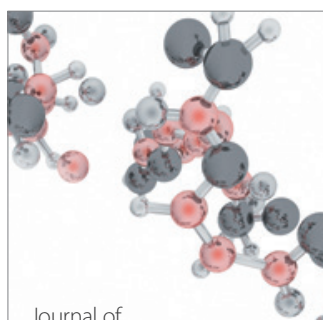

Analytical Methods

in Chemistry

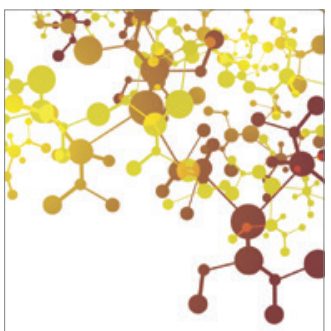

Journal of

Applied Chemistry

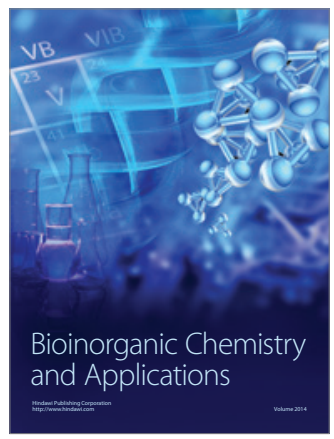

Inorganic Chemistry
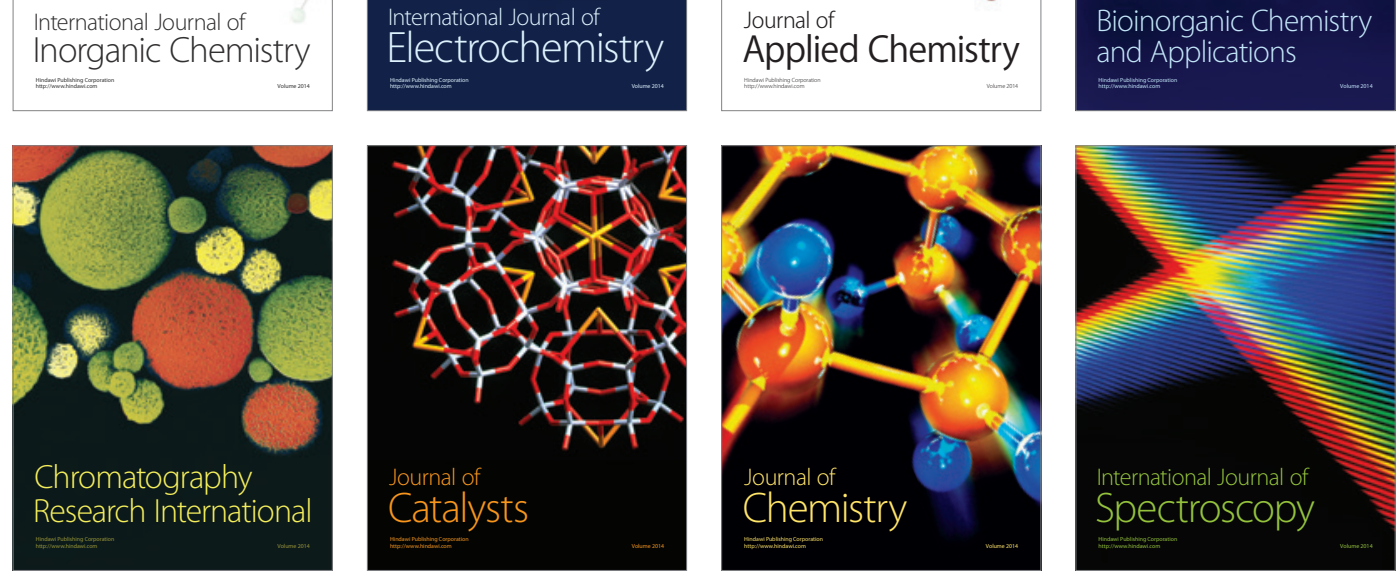